\title{
Immunohistochemical Study of Non-Epithelial Cells in Spiradenoma
}

\author{
Keigo Ito ${ }^{1,2}$, Shin-ichi Ansai ${ }^{1}$ and Hidehisa Saeki ${ }^{3}$ \\ ${ }^{1}$ Division of Dermatology and Dermatopathology, Nippon Medical School Musashi Kosugi Hospital, Kanagawa, Japan \\ ${ }^{2}$ Department of Dermatology, Jikei University School of Medicine, Tokyo, Japan \\ ${ }^{3}$ Department of Dermatology, Nippon Medical School, Tokyo, Japan
}

Background: Intratumoral lymphocytes are a defining feature of spiradenoma; however, there have only been a few reports on the phenotypic features of non-epithelial cells. Spiradenomas also contain numerous cells positive for S-100 protein and the nature of these cells is still controversial.

Methods: We performed a clinicopathological and immunohistochemical study of ten cases of spiradenoma.

Results: The study included seven men and three women. On histopathological examination, spiradenoma could be divided into two types: the vascular proliferating (VP) type (five cases) that featured granulation tissue with edema, vascular proliferation, and inflammatory cell infiltration into the stroma, and the common type (five cases), which did not include any of the aforementioned features. Immunohistochemical staining demonstrated a large number of cells positive for S-100 protein. These included cells with large pale nuclei, dendritic cells, and a few cells with small dark nuclei that were also positive for $\alpha$-smooth muscle actin. Most of the cells infiltrating the parenchymata of these lesions were CD3-positive. The proportions of CD4-positive and CD8-positive cells were almost equal or CD8positive cells were predominant. CD20+ cells were observed in five spiradenomas. In painful lesions, there were numerous nerve fibers near the tumor.

Conclusions: In spiradenoma, CD3 + T cells were mainly seen in the parenchyma and CD8+ cells were predominant over CD4+ cells in most cases. CD20+ cells showed focal infiltration of the parenchyma and stroma, especially in VP-type lesions. S-100 protein-positive cells in spiradenoma contained not only Langerhans cells, but also cells with myoepithelial differentiation.

(J Nippon Med Sch 2019; 86: 15-21)

Key words: spiradenoma, vascular proliferating type, T lymphocyte, myoepithelial cell

\section{Introduction}

Spiradenoma is a benign tumor that presents clinically as a painful, slightly bluish, intradermal or subcutaneous nodule, and was first reported by Kersting \& Helwig ${ }^{1}$. Histological examination reveals epithelial cells arranged in intertwining bands, and two types of cells can be recognized. Cells with small dark nuclei are present at the periphery of the bands, whereas cells with large pale nuclei form small lumina at the center of the bands ${ }^{1-4}$. Spiradenoma are divided into two subtypes, the common type and the vascular proliferating type that features proliferation of blood vessels, hemorrhage, edematous stroma, and sometimes granulation tissue ${ }^{5}$.
The most characteristic histopathological feature of spiradenoma is infiltration of non-epithelial cells, including Langerhans cells and $\mathrm{T}$ lymphocytes, into the parenchyma. However, there have only been a few reports on the phenotypic features of non-epithelial cells in the parenchyma and stroma of spiradenoma based on a relatively small number of cases ${ }^{6,7}$. We performed the present study to investigate the detailed phenotypic features of the cells infiltrating spiradenomas and compared lesions of the common type and the vascular proliferating type.

Spiradenomas also contain numerous cells positive for S-100 protein and the nature of these cells is still controversial, so we investigated the S-100-positive cells in this

Correspondence to Shin-ichi Ansai, MD, PhD, Division of Dermatology and Dermatopathology, Nippon Medical School

Musashi Kosugi Hospital, 1-396 Kosugi-cho, Nakahara-ku, Kawasaki, Kanagawa 211-8533, Japan

E-mail: shin8113@nms.ac.jp

Journal Website (http://www2.nms.ac.jp/jnms/) 
study.

Spiradenoma is known as a painful tumor, but the relationship between the distribution of nerve fibers and pain is still controversial. We retrieved nerve fibers near the tumor and examined the histopathological and immunohistochemical relationship.

\section{Materials and Methods}

\section{Patients and Specimens}

Slides with routine HE staining were obtained from ten patients treated at the Department of Dermatology of Jikei University School of Medicine Hospital over the past 16 years (1993-2009). Clinical information on the patients, including complaints of pain, is provided in Table 1.

\section{Histopathology}

A histopathological diagnosis of spiradenoma was made according to the published criteria ${ }^{2,3}$. Briefly, the lesions were composed of one or a few nodules of basaloid cells in the dermis and/or subcutaneous tissue and were round with smooth borders at a low magnification. At a higher magnification, two distinct populations of neoplastic cells were recognized, i.e., cells with small dark nuclei and cells with large pale nuclei. These cells were arranged in intertwining bands, with the cells having small dark nuclei at the periphery of the bands and the cells with large pale nuclei forming small lumina at the center of the bands. The lesions were divided into two subtypes, the vascular proliferating (VP) type of spiradenoma with proliferation of blood vessels, hemorrhage, edematous stroma, and sometimes granulation tissue, and the common type without such findings.

\section{Immunohistochemistry}

Immunohistochemistry was performed using the amino acid polymer method (Nichirei, Tokyo, Japan). For antigen retrieval, paraffin-embedded sections were incubated for $45 \mathrm{~min}$ at $98^{\circ} \mathrm{C}$ in $1 \mathrm{mmol} / \mathrm{L}$ ethylenediamine tetraacetic acid and $10 \mathrm{mmol} / \mathrm{L}$ Tris-HCL buffer ( $\mathrm{pH}$ 9.0). Primary antibodies directed against CD3 (Novocastra; 1: 100), CD4 (Novocastra; 1:50), CD8 (Novocastra; 1:100), CD20 (Dako Japan; 1:100), CD56 (Novocastra; 1:100), S100 protein (Dako Japan; 1:400), and $\alpha$-smooth muscle actin (SMA) (Dako Japan; 1:100) were prepared and sections were incubated with the primary antibodies overnight at $4{ }^{\circ} \mathrm{C}$ in a moist chamber. Next, the sections were incubated with Simple Stain MAX-PO (Nichirei) for 30 min and peroxidase binding sites were revealed by using 3,3'-diaminobenzidine-tetrachloride (Dojindo, Kumamoto, Japan). After that, the sections were counterstained and mounted.

Expression of CD3, CD4, CD8, CD20, and CD56 by lymphoid cells infiltrating into the parenchyma and stroma was assessed semiquantitatively using three grades, -: negative, \pm : a few positive cells (less than 20\% of the cells were positive), and +: many positive cells (more than $20 \%$ of the cells were positive). We also compared the proportions of cells positive for CD4 and CD8.

\section{Results}

Clinical and immunohistochemical findings of the ten patients are summarized in Table $\mathbf{1 .}$

\section{Clinical Findings}

There were seven men and three women, with a mean age at resection of $54.8(44-73)$ years. Four lesions (40\%) were located on the trunk, two lesions each $(20 \%)$ on the face and upper extremities, and one lesion each (10\%) on the scalp and neck. Nine patients had solitary lesions and one had multiple lesions. Seven of the ten patients clinically complained of pain, whereas one did not and its presence or absence in the other two was unknown.

\section{Histopathological Findings}

Five lesions (two each on the face and trunk and one on the hand) were the common type of spiradenoma (Fig. 1) and the other five lesions (two on the trunk and one each on the neck, arm, and scalp) were the VP type of spiradenoma (Fig. 2). Nerves were found in the stromata near tumor cell nests in eight of the ten cases (80\%) (Fig. 3). In seven of those eight cases, the patients complained of pain, whereas it was unknown whether the other patient had such a complaint.

\section{Immunohistochemical Findings}

\section{CD3, CD4, and CD8}

CD3-positive lymphocytes were observed in both the parenchyma and the stroma, although the number of such cells was usually larger in the parenchyma (Fig. 4). In two cases, few or no CD3+ lymphocytes were observed. In the VP type of spiradenoma, a similar number of these cells existed in the stroma and parenchyma.

CD4+ lymphocytes were observed in seven lesions, whereas the other three lesions had few or none (Fig. 5a). CD4+ lymphocytes were more frequent in the parenchyma than in the stroma. The distribution of CD8+ lymphocytes was similar to that of CD4+ lymphocytes. There were more CD8+ lymphocytes than CD4+ lymphocytes infiltrating the parenchymata in six lesions (60\%) (Fig. 5 b), but these cells showed almost equal numbers in the other four lesions. In one lesion (case 6) with fibrosing granulation tissue due to rupture of a cystic area, there 


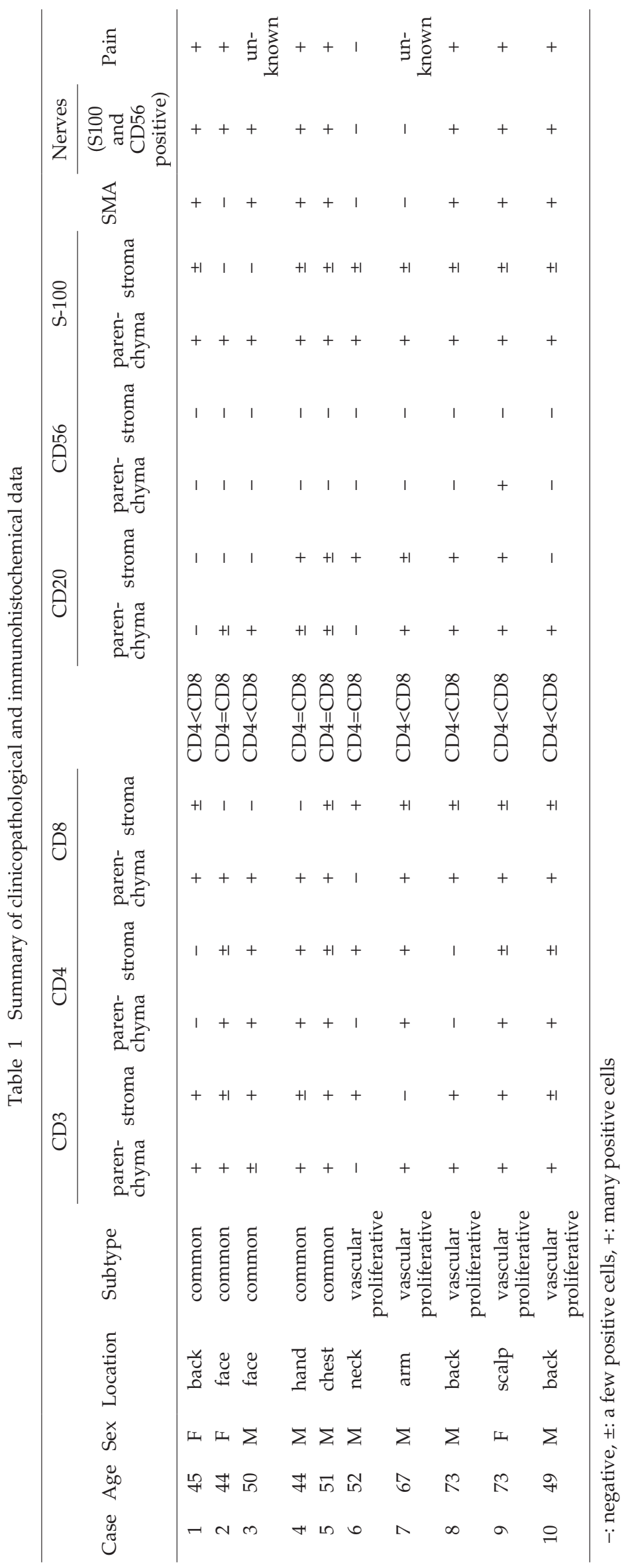




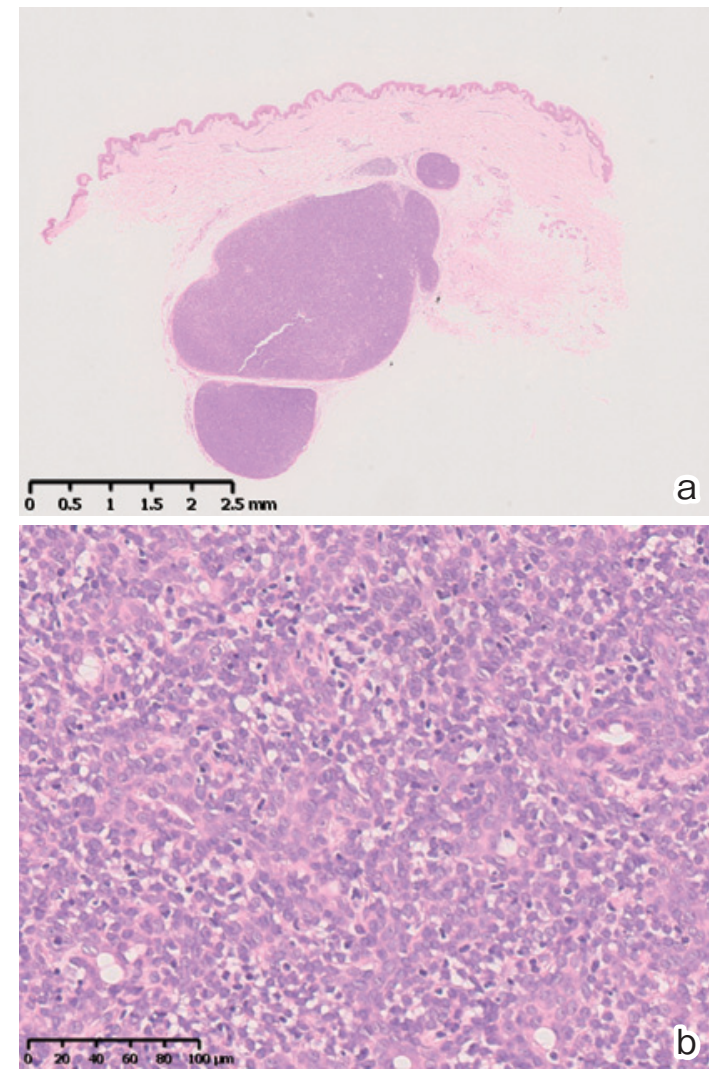

Fig. 1 Histopathological features of the common type of spiradenoma. The lesion is composed of a few round nodules of basaloid cells with smooth borders at low magnification in the dermis and subcutaneous tissue. (a) At a higher magnification, two distinct populations of neoplastic cells can be seen: cells with small dark nuclei and cells with large pale nuclei. These cells are arranged in intertwining bands. The cells with small dark nuclei are present at the periphery of the bands, whereas those with large pale nuclei form small lumina in the center of the bands. (b)

$\mathrm{H} \& \mathrm{E}$ stain

were almost no lymphocytes infiltrating the parenchyma, but some lymphocytes were seen in the stroma (Fig. 6).

CD20

In five lesions (50\%), CD20+ lymphocytes mainly showed focal infiltration of the parenchymata (Fig. 7). CD20+ lymphocytes were more abundant in the VP type of spiradenoma than in the common type.

\section{CD56}

In almost all of the lesions, no CD56+ lymphocytes were seen in either the parenchyma or stroma. However, CD56-positive nerves were observed in the stromata near tumor cell nests in eight of the ten patients $(80 \%)$.

S-100 protein

S-100 protein-positive cells were observed in the parenchymata of all lesions (Fig. 8), whereas few S-100-protein

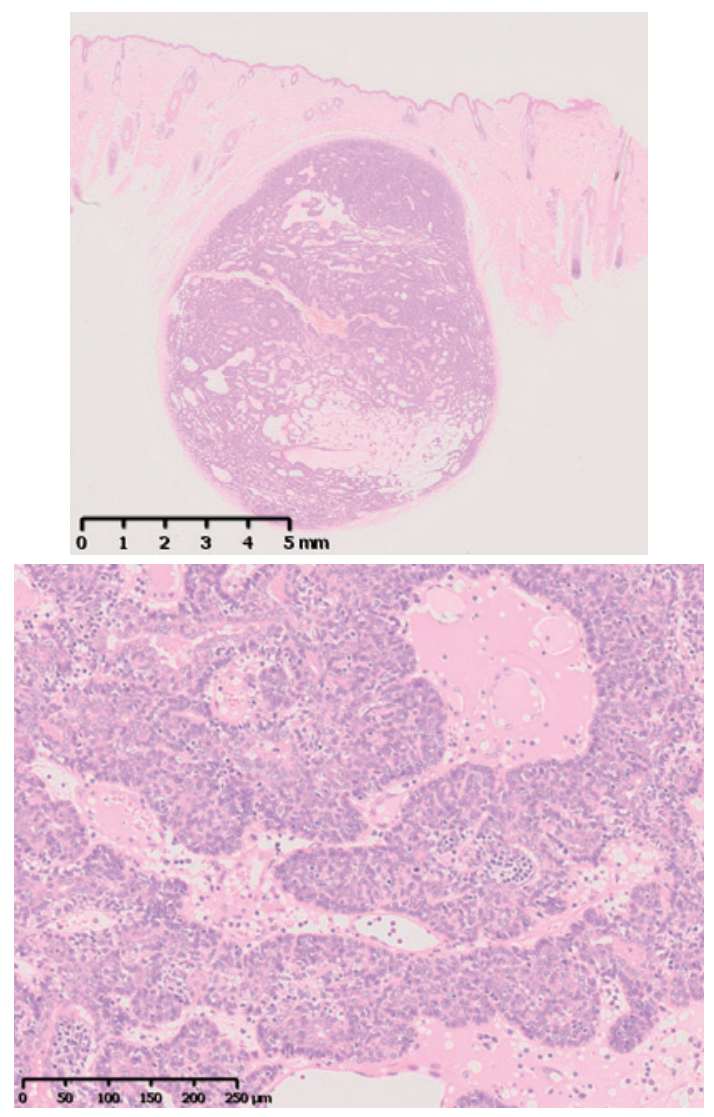

Fig. 2 Histopathological features of the vascular proliferating (VP) type of spiradenoma. Proliferation of blood vessels, hemorrhage, edematous stroma, and granulation tissue are observed. $\mathrm{H} \& \mathrm{E}$ stain

positive cells were seen in the stroma (Fig. 8). S-100 protein-positive cells mainly had large pale nuclei, although dendritic cells were also positive and showed stronger staining. Only a few of the cells with small dark nuclei were positive for S-100 protein. Numerous S-100positive nerves were observed in the stromata near tumor cell nests in eight of the ten patients (80\%) (Fig. 3b).

\section{SMA}

SMA-positive cells were also seen in seven of the ten cases (Fig. 9). These cells generally had small dark nuclei.

\section{Discussion}

This study revealed that the spiradenomas in our patient population contained numerous cells positive for S-100 protein, particularly cells with large pale nuclei and dendritic cells. A few of the cells with small dark nuclei were also positive for S-100 protein. As reported by lida and colleagues, the dendritic cells in spiradenoma are thought to be Langerhans cells ${ }^{7}$. While the role of Langerhans cells in spiradenoma is not clear, these cells might act as part of the immune surveillance system, resulting in the benign nature of this tumor, as suggested by lida et $\mathrm{al}^{7}$. 

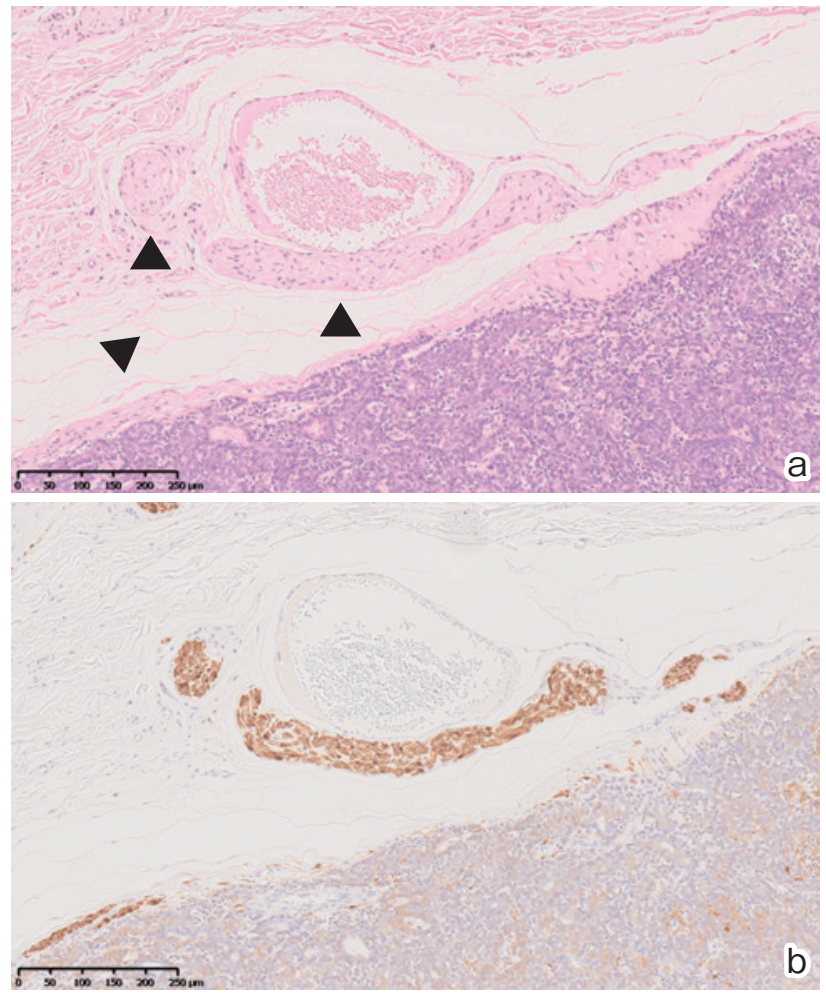

Fig. 3 Nerve fibers are observed near a lesion. (arrowhead: a) The nerve fibers are highlighted by immunohistochemical staining using an anti-S-100 protein antibody. (b)

a: $\mathrm{H}$ \& E stain, b: immunoperoxidase stain for S-100 protein

While S-100 protein is used as a marker of Langerhans cells, it can also be expressed by the secretory cells of eccrine sweat glands and myoepithelial cells. Some of the cells with small dark nuclei not only expressed S-100 protein but also SMA, as reported previously ${ }^{8-10}$. Therefore, these cells showed at least some degree of myoepithelial differentiation, because neoplastic cells do not always express antigens in the same way as normal cells. On the other hand, the status of the S-100-positive cells with large pale nuclei is still controversial. These cells might show differentiation towards the secretory cells of eccrine sweat glands ${ }^{11}$, but most dermatopathologists think that spiradenoma is an apocrine tumor for several reasons ${ }^{3,4}$.

We divided the spiradenomas into two different subtypes (a common type and a VP type) according to a previously published report ${ }^{5}$. Histopathologically, the VP type shows proliferation of blood vessels, hemorrhage, edematous stroma, and sometimes granulation tissue, whereas the common type does not have these findings. Ansai et al. reported that spiradenomas of the VP type were older than those of the common type ${ }^{5}$. They also re-

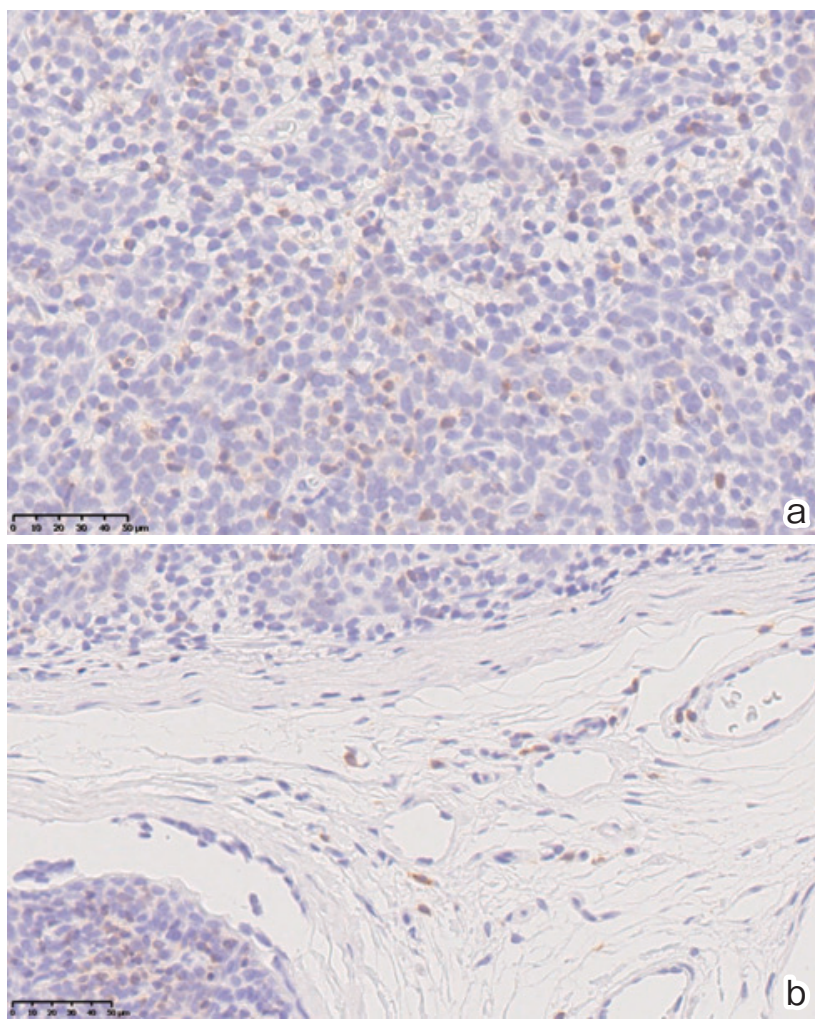

Fig. 4 CD3+ cells infiltrating the parenchyma (a) and the stroma (b) of a lesion. Immunoperoxidase stain for CD3.

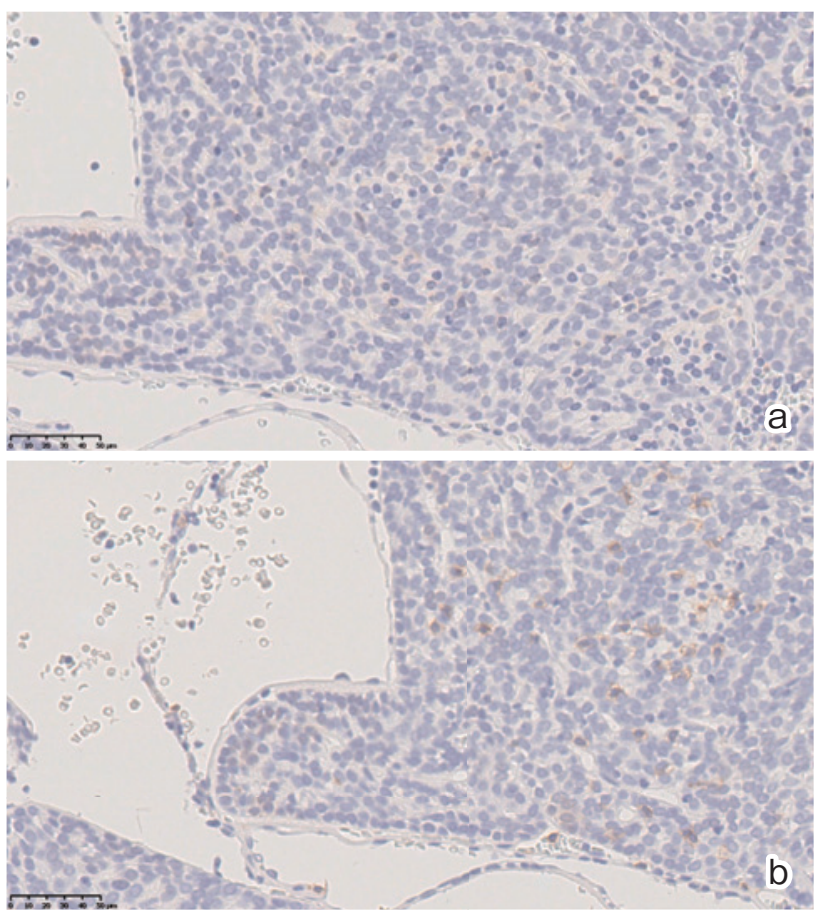

Fig. 5 More CD8+ cells than CD4+ cells are observed in the parenchyma.

a: immunoperoxidase stain for CD4, b: immunoperoxidase stain for $\mathrm{CD} 8$ 


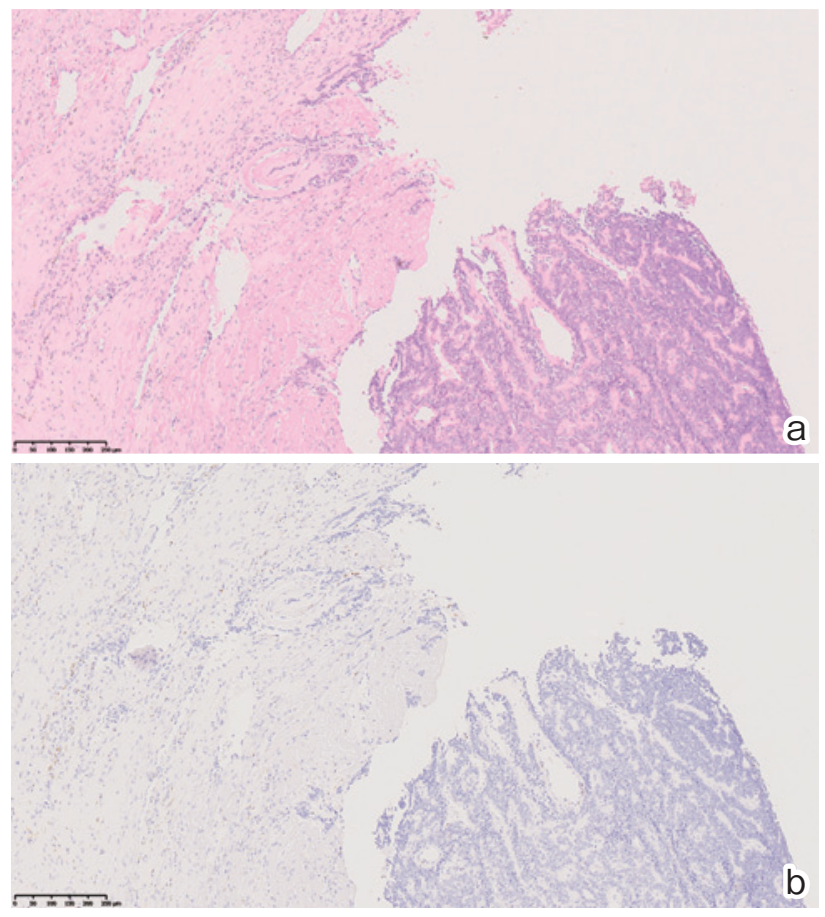

Fig. 6 In one lesion with fibrosing granulation tissue, there are almost no lymphocytes in the parenchy$\mathrm{ma}$, but some lymphocytes can be seen in the stroma.

a: H \& E stain, b: immunoperoxidase stain for CD3

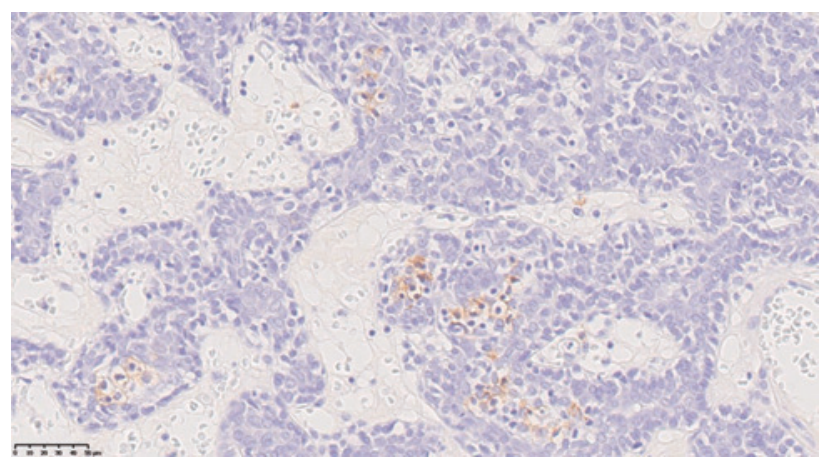

Fig. 7 CD20+ lymphocytes mainly show focal infiltration of the parenchyma. Immunoperoxidase stain for CD20.

ported that $43.1 \%$ of spiradenomas corresponded to the VP type ${ }^{5}$. Their findings were similar to ours in this study.

Intratumoral lymphocytes are a defining feature of spiradenoma ${ }^{3}$, but the phenotypes of infiltrating lymphocytes have not been addressed, except in a few reports ${ }^{6,7}$. We demonstrated that a large number of non-epithelial cells were distributed evenly throughout the parenchymata of the spiradenomas in this series, consisting primarily of S-100+ Langerhans cells, CD3+ T cells, and a small number of CD20+ B cells. As already reported, the

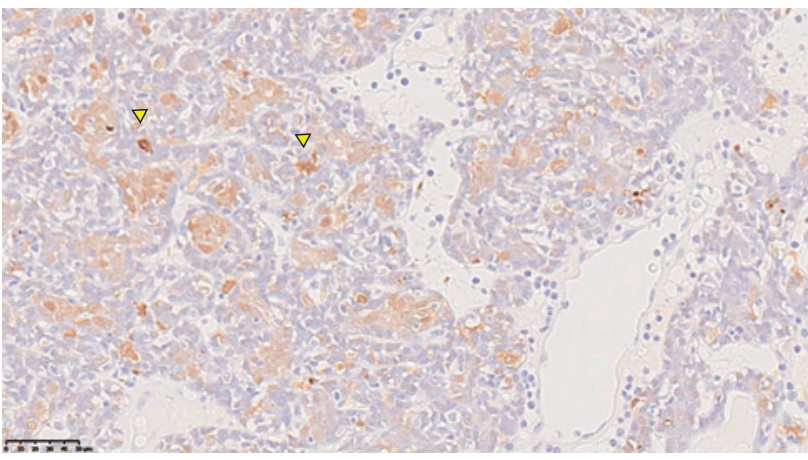

Fig. 8 S-100-positive cells can be observed in the parenchyma, whereas there are few S-100-positive cells in the stroma. S-100 protein is mainly positive in cells with large pale nuclei, but is also positive in dendritic cells (arrowhead). Immunoperoxidase staining for S-100 protein.

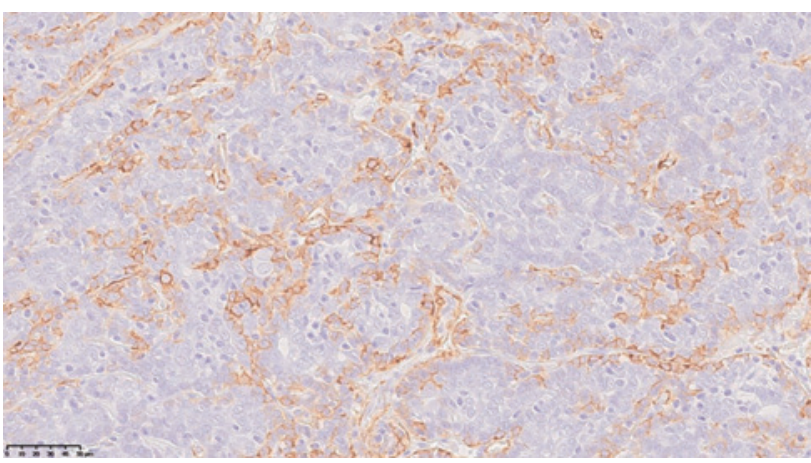

Fig. 9 SMA is mainly expressed in the cells with small dark nuclei. Immunoperoxidase stain for $\alpha$-smooth muscle actin.

proportions of CD4 and CD8 cells in the parenchyma were almost equal or CD8 cells were predominant ${ }^{7}$. We also compared the proportions of these cells in common spiradenomas and VP spiradenomas, but there were no marked differences between the two subtypes. Interestingly, in a spiradenoma with fibrosis and granulation tissue, very few lymphocytes infiltrated the parenchyma. This finding suggested that intratumoral lymphocytes, especially CD3+ T cells, might disappear after tissue disturbance. However, we only observed one such lesion, so further investigation is required to determine the frequency of such findings.

The distribution of CD20+ B cells in spiradenoma has rarely been mentioned in other reports ${ }^{6,7}$. In this study, focal infiltration of these cells was seen in some cases. These cells were more abundant in both the parenchyma and stroma of VP spiradenomas than in common-type spiradenomas. From these findings, we speculated that B lymphocytes might play an important role in granulation 
tissue of VP-type lesions, but the detailed role of those cells is currently unclear. We also examined the distribution of CD56+ cells. Similar to the finding reported by Iida et $\mathrm{al}^{7}{ }^{7}$, almost no CD56+ cells were detected in our 10 spiradenomas.

Spiradenoma is often a painful tumor. It has been demonstrated that nerve fibers are abundant near spiradenomas ${ }^{6}$. We also found abundant nerve fibers by immunohistochemistry for S-100 protein and CD56 in eight of the ten cases. The patient histologically lacking nerves did not complain of pain, whereas painful lesions exhibited numerous nerve fibers near the tumor. From these findings, we speculated that the number of nerve fibers near the tumor was related to the pain of the lesion.

We also found that in two out of three SMA-negative cases, nerve fibers were not observed. This phenomenon may indicate that proliferation of nerve fibers is less likely to occur in the cases lacking myoepithelial differentiation. Furthermore, both cases histologically lacking nerves were the VP type in this study. We cannot confirm whether such a phenomenon is specific for VP type lesions. There has been no report outlining the relationship between pain of the lesions and histological subtypes. Further examination is required to confirm whether such a phenomenon exists.

In summary, we investigated the phenotypic features of neoplastic and non-epithelial cells in spiradenoma by immunohistochemistry. Some of the cells with small dark nuclei were positive for both S-100 and SMA, suggesting at least some degree of myoepithelial differentiation, but the nature of the cells with large pale nuclei remains controversial. S-100-positive Langerhans cells were abundant in the parenchyma, but few such cells existed in the stroma. CD3+ $\mathrm{T}$ cells were mainly seen in the parenchyma and CD8+ cells were predominant over CD4+ cells in most cases. CD20+ cells showed focal infiltration of the parenchyma and stroma, whereas CD56+ cells were almost completely absent. Finally, nerve fibers were abundant near the tumor cell nests, especially in painful lesions.

Conflict of Interest: None declared.

\section{References}

1. Kersting E, Helwig EB: Eccrine spiradenoma. Arch Dermatol 1956; 73: 199-227.

2. Mambo NC: Eccrine spiradenoma: clinical and pathologic study of 49 tumors. J Cutan Pathol 1983; 10: 312-332.

3. Requena L, Kiryu H, Ackerman AB: Spiradenoma. In Neoplasms with Apocrine Dfferentiation, 1998; pp 469513, Lippincott-Raven Publishers, Philadelphia.

4. Kazakov DV, Michal M, Kacerovska D, McKee PH: Spiradenoma, Cylindroma, Spiradenocylindroma. In Cutaneous Adnexal Tumors, 2012; pp 40-59, Wolters Kluwer Health/Lippincott Williams \& Wilkins, Philadelphia.

5. Ansai S, Anan T, Fukumoto T, Kimura T: A clinicopathological study of spiradenoma. Jpn J Dermatol 2013; 123: 1505-1513(in Japanese).

6. Al-Nafussi A, Blessing K, Rahily M: Non-epithelial cellular components in eccrine spiradenoma: a histological and immunohistochemical study of 20 cases. Histopathology 1991; 18: 155-160.

7. Iida K, Iwai S, Hosaka H, Kitami Y, Akiyama M, Suzuki $\mathrm{T}$, Sueki H: Immunohistochemical characterization of nonepithelial cells in spiradenoma. J Dermatol 2013; 40: 896900.

8. Eckert F, Betke M, Schmorckel C, Neuwiler J, Schmid U: Myoepithelial differentiation in benign sweat gland tumors. J Cutan Pathol 1992; 19: 294-301.

9. Wiley EH, Milchgrub S, Freeman RG, Kim E: Sweat gland adenomas; immunohistochemical study with emphasis on myoepithelial differentiation. J Cutan Pathol 1993; 20: 337-343.

10. Meybehm M, Fischer HP: Spiradenoma and dermal cylindroma: Comparative immunohistochemical analysis and histogenetic considerations. Am J Dermatopathol 1997; 19: 154-161.

11. Pennys NS: Immunohistochemistry of adnexal neoplasms. J Cutan Pathol 1984; 11: 357-364.

(Received, April 2, 2018)

(Accepted, September 21, 2018) 